RESEARCH PAPER RP1358

Part of Journal of Research of the National Bureau of Standards, Volume 26, January 1941

\title{
EFFECT OF HEAT TREATMENT AND COOLING RATE ON THE MICROSCOPIC STRUCTURE OF PORTLAND CEMENT CLINKER
}

\author{
By George W. Ward
}

\section{ABSTRACT}

Ten commercial portland cement clinkers with their slowly and quickly cooled counterparts were examined microscopically. Methods are given for the conduction of the microscopical examination.

The appearance of the clinker phases after the different heat treatments is described and the structure of the clinkers discussed.

In general, for all clinkers, the phase compositions as calculated with consideration of glass agreed better with the microscopically determined phases than did those based upon the calculations in which complete equilibrium crystallization was assumed. The microscopically determined $3 \mathrm{CaO} . \mathrm{SiO}_{2}$ was nearly always greater and the $2 \mathrm{CaO} . \mathrm{SiO}_{2}$ lower than that calculated by either of the other methods. The $3 \mathrm{CaO} \cdot \mathrm{Al}_{2} \mathrm{O}_{3}$ was always less than that calculated from chemical analyses. Generally the $4 \mathrm{CaO} \cdot \mathrm{Al}_{2} \mathrm{O}_{3} \cdot \mathrm{Fe}_{2} \mathrm{O}_{3}$ found microscopically exceeded that calculated by either of the other methods. The glass determined microscopically and the values obtained from the heat of solution method agreed better for slowly and plant cooled than for quickly cooled clinkers.

\section{CONTENTS}

I. Introduction

1. Heat treatment

2. Methods of examination

II. Description of clinker minerals

1. Free lime _.

2. Magnesia

3. Tricalcium silicate

4. Dicalcium silicate

5. Dark interstitial material

6. Tetracalcium aluminoferrite

III. Comparison of phase compositions as determined microscopically and as calculated from analytical data

IV. Summary

V. References

\section{INTRODUCTION}

Investigators have recognized that the relative proportions of the phases in portland cement clinker exert an important influence on the properties of the resulting concrete. In recent years successful attempts have been made to vary the relative proportions of the constituents by changing the cooling rate of clinker [1]. ${ }^{1}$ Correlations between calculated clinker compositions assuming complete crystallization and the physical properties of the resulting cements have not

\footnotetext{
1 Figures in brackets indicate the literature references at the end of this paper.
} 
been precise. Comparisons between calculated and microscopically determined phase compositions of commercial clinkers have been published recently [2].

In the present report data are presented upon variously heattreated clinkers as well as the plant products. New information was gained on the microscopic structure of portland cement clinker. The effects of heat treatment, particularly the rate of cooling, on the phases present are described. Correlations are made between observed phases and calculated phase compositions.

\section{HEAT TREATMENT}

Ten samples were taken from a series of 26 commercial clinkers which covered the range of compositions found in commercial practice. One portion of each clinker, designated as $P$ or "plant" clinker, was examined in its original condition. A second portion of each clinker was reheated in an experimental rotary kiln ( 5 in. by $8 \mathrm{ft}$ ) at a temperature of $1,400^{\circ}$ to $1,425^{\circ} \mathrm{C}\left(2,552^{\circ}\right.$ to $\left.2,597^{\circ} \mathrm{F}\right)$ and cooled to $1,250^{\circ} \mathrm{C}\left(2,282^{\circ} \mathrm{F}\right)$ in 2 to 3 minutes in order to yield a maximum amount of glass. This quickly cooled clinker was designated as $Q$. A third portion was reheated to the same temperature in a laboratory batch kiln and cooled to $1,250^{\circ} \mathrm{C}\left(2,282^{\circ} \mathrm{F}\right)$ at a uniform rate in about 3 hours in order to yield a minimum of glass. This slowly cooled clinker was designated as $S$. The heating and cooling were carried out under oxidizing conditions. The 10 random clinkers with their variously heat-treated counterparts gave 30 samples for study.

\section{METHODS OF EXAMINATION}

The clinkers were studied microscopically and were analyzed chemically. ${ }^{2}$ The investigation with the microscope included examination of powders, thin [3] and polished thin sections [4], together with variously etched polished specimens $[4,5,6]$.

Quantitative measurements were made using etched, polished specimens suitably mounted in Bakelite resin [6]. When ready for examination the surface of each specimen contained from 15 to 20 clinker particles. All quantitative measurements were made with a Wentworth [7] micrometer attached to the microscope stage. Each value reported was the average of five equally spaced traverses on each of five mounts.

The etchants proposed by Tavasci [4] and Insley [5], with modifications in some instances, were found to be generally satisfactory.

Glass was determined microscopically after a water etch followed by 1-percent nitricacid-alcohol solution, and after an etch by 10-percent potassium hydroxide in water [8].

\section{DESCRIPTION OF CLINKER MINERALS}

The various clinker phases are described below as they appeared in etched polished specimens when viewed microscopically by reflected light. Figures 1,2 , and 3 , although used to illustrate the typical appearance of all clinkers after the various heat treatments, represent

\footnotetext{
2 Analyses by C. L. Ford, Portland Cement Association, Chicago, Ill.
} 


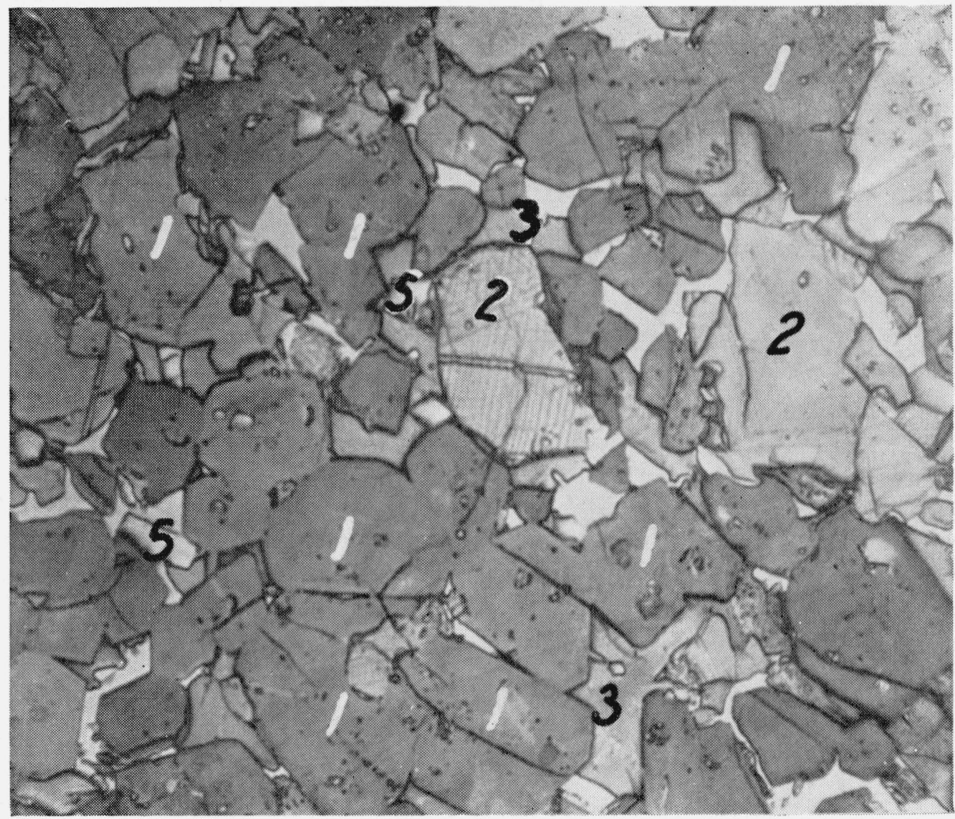

Figure 1.-Typical slowly cooled clinker.

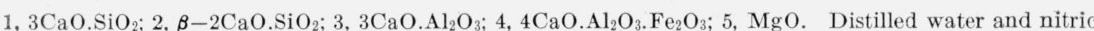
acid etch. Magnification $\times 500$. Reflected light. 


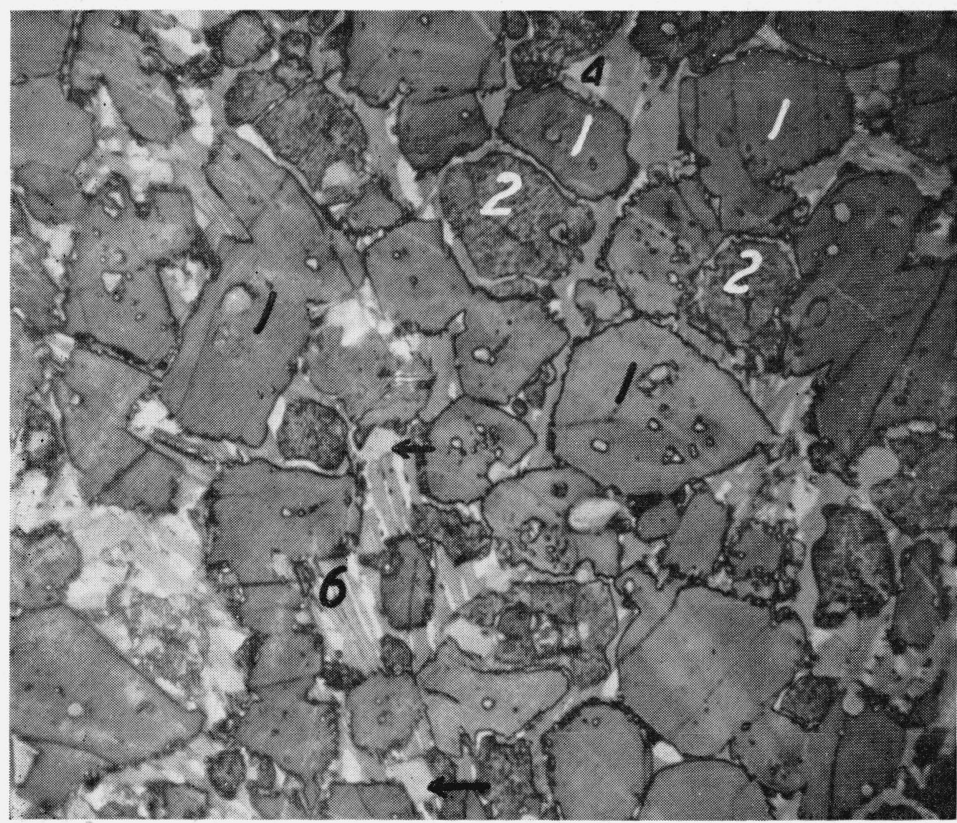

FIGURE 2.-Typical plant-cooled clinker.

1, $3 \mathrm{CaO} . \mathrm{SiO}_{2} ; 2, \boldsymbol{\beta}-2 \mathrm{CaO} . \mathrm{SiO}_{2} ; 4,4 \mathrm{CaO} . \mathrm{Al}_{2} \mathrm{O}_{3} . \mathrm{Fe}_{2} \mathrm{O}_{3} ; 6$, prismatic dark interstitial material, arrows indicate unknown phases. Note $\boldsymbol{\beta}-2 \mathrm{CaO} . \mathrm{SiO}_{2}$ rims around $3 \mathrm{CaO} . \mathrm{SiO}_{2}$. Distilled water and nitric acid etch. Magnification $\times 500$. Reflected light. 


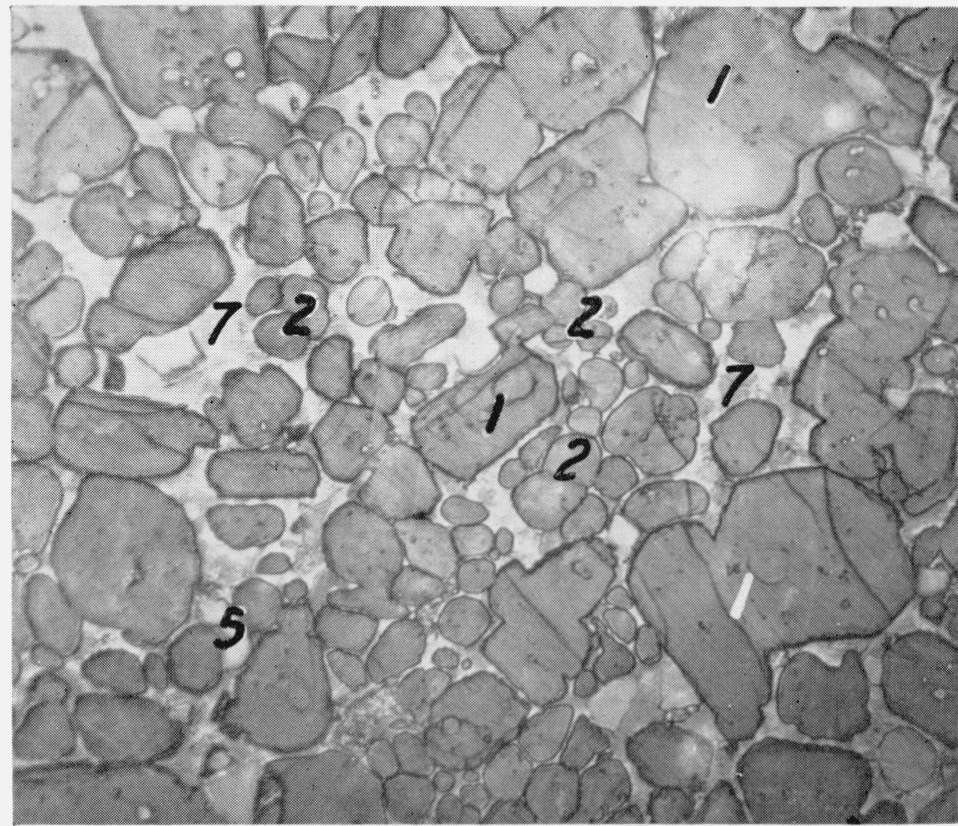

Figure 3.-Typical quickly cooled clinker.

1, $3 \mathrm{CaO} . \mathrm{SiO}_{2} ; 2, \beta-2 \mathrm{CaO} . \mathrm{SiO}_{2} ; 5, \mathrm{MgO} ; 7$, glass and light interstitial material, $4 \mathrm{CaO} . \mathrm{Al}_{2} \mathrm{O}_{3} . \mathrm{Fe}_{2} \mathrm{O}_{3} . \quad$ Distilled water and nitric acid etch. Magnification $\times 500$. Reflected light.
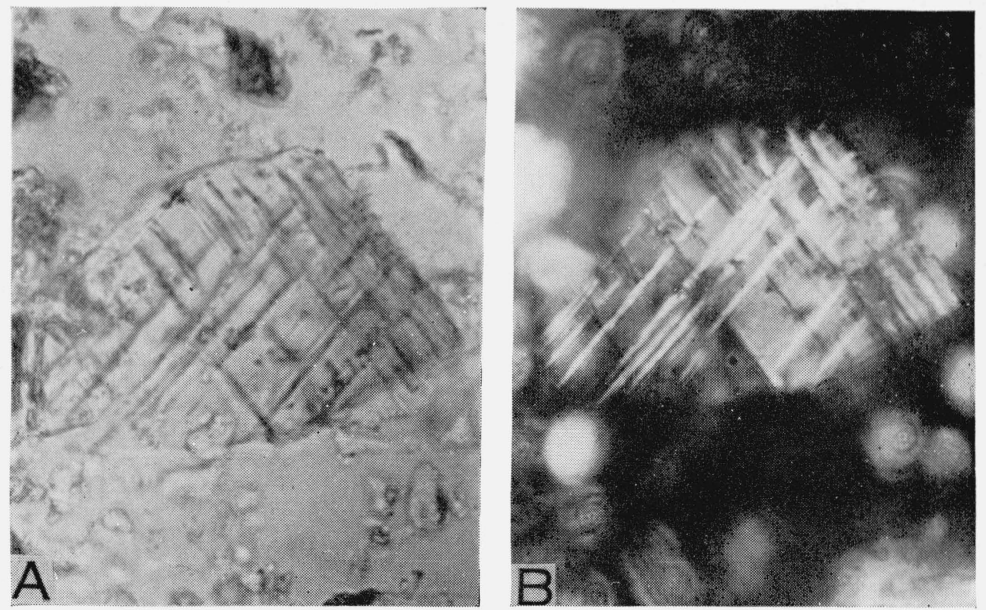

Figure 4.-3CaO. $\mathrm{SiO}_{2}$ in transmitted light, twinning simulated by unknown inclusions.

$A$, Uncrossed Nicols; $B$, crossed Nicols. Magnification $\times 1000$. Transmitted light. 
specifically the changes effected in sample 3 by slow, plant, and quick cooling, respectively.

\section{FREE LIME}

Free lime $(\mathrm{CaO})$ etched readily after 1 minute's exposure in 1-3 water-alcohol. It appeared as dark gray, almost black, rounded, or elliptical grains with no zoning, twinning, or inclusions. It occurred as inclusions in $3 \mathrm{CaO} . \mathrm{SiO}_{2}$ and in the interstitial material. As the cooling became slower the amount of free lime decreased.

In general, free lime was the first phase to crystallize. However in slowly cooled clinkers, where the cooling occurred at a uniform rate from $1,450^{\circ}$ to $1,250^{\circ} \mathrm{C}$ in about 3 hours, some of the free lime crystallized after $3 \mathrm{CaO} \cdot \mathrm{SiO}_{2}$.

\section{MAGNESIA}

Magnesia, or periclase ( $\mathrm{MgO})$, was observed without etching as brilliant crystals of high relief occurring well distributed throughout the interstitial material and occasionally in slowly cooled and plantcooled clinkers, as inclusions in $3 \mathrm{CaO}$. SiO ${ }_{2}$. As the cooling became slower crystal form became more prominent, octahedrons being found in slowly cooled clinker. Cracking around the rims of the grains almost always occurred, but it tended to decrease in quickly cooled clinkers, possibly because of the small crystal size in that type of clinker. By actual measurement of many periclase particles, their average size was found to be greater in slowly cooled than in plantcooled clinkers, and larger in plant-cooled than in quickly cooled clinkers.

\section{TRICALCIUM SILICATE}

Tricalcium silicate $\left(3 \mathrm{CaO} . \mathrm{SiO}_{2}\right)$ etched as dark gray, almost black, crystals after 3 to 5 seconds agitation in 1-percent $\mathrm{HNO}_{3}$-alcohol mixture. Probably because of difference in orientation, a few crystals etched to a lighter color. It was commonly the most abundant phase. In slowly cooled clinkers it tended to be completely or partially included in tricalcium aluminate crystals. The development of good crystal form was observed to decrease with increased rate of cooling.

Re-solution ${ }^{3}$ of $3 \mathrm{CaO} \cdot \mathrm{SiO}_{2}$ and the resulting precipitation of $\beta 2 \mathrm{CaO} . \mathrm{SiO}_{2}$ were more prominent in slowly cooled than in plantcooled clinkers and only very limited in quickly cooled clinkers. This re-solution was indicated by embayed tricalcium silicate with dicalcium silicate occupying the bays and frequently by fringes of dicalcium silicate around tricalcium silicate. The particularly good examples of partial and complete pseudomorphs of dicalcium silicate after tricalcium silicate in specimens $4 P$ and $7 P$ were other indications of re-solution.

The tendency to form twins or to be zoned decreased as the cooling became more rapid. Good examples of simple twinning were found in slowly cooled and plant-cooled clinkers with a greater number in the former. Zoning was well developed in the slowly cooled clinker, with three or four zones not unusual, but it was developed to a lesser extent in the plant-cooled product and was rarely observed in quickly cooled samples.

3 L. S. Brown considers this as dissociation; see "Tricalcium Aluminate and the Microstructure of Portland Cement Clinker." Proc. Am. Soc. Testing Materials 37, pt. 2, 279 (1937). 
Inclusions of dicalcium silicate, magnesia, and free lime, sometimes accompanied by tetracalcium aluminoferrite, frequently occurred in tricalcium silicate irrespective of heat treatments. In a few instances periclase inclusions had dark halos, possibly indicative of solution in tricalcium silicate. In clinkers $4 P$ and $4 Q$ a special type of inclusion arranged to simulate complex twinning, as seen in dicalcium silicate, was noted. Because of their small size, it has not been possible to identify these inclusions, although they resemble in arrangement and general appearance the crystallization of calcium ferrite from tricalcium silicate found by Anderson and Lee [9] in slag. This phenomenon is illustrated in figure 4.

\section{DiCALCIUM SILICATE}

Dicalcium silicate $\left(2 \mathrm{CaO} \cdot \mathrm{SiO}_{2}\right) . \quad \beta$-Dicalcium silicate appeared without good crystal form, generally as spherical or elliptical crystals, which etched readily in 1-percent nitric-acid-alcohol solution. Their distribution was generally good, but in some clinkers there was a strong tendency to form large groups. Commonly $\beta$-dicalcium silicate occurred closely associated with tricalcium silicate as fringes, as inclusions, as pseudomorphs, or occupying embayments.

In quickly cooled clinkers, dicalcium silicate with complex twinning was the most frequently observed form of this phase. Occasionally free lime was found as inclusions, and tetracalcium aluminoferrite appeared along twinning planes and in cracks. Simplicity of form, a general lack of inclusions, and fewer irregularities characterized the dicalcium silicate of quickly cooled clinkers.

In slowly cooled clinkers, dicalcium silicate untwinned and with simple multiple twinning was more prominent. Frequently twinning extended out past the usually rounded-particle boundary to give a many-fingered appearance to the grain. It was not unusual for twinning to be indicated by an arrangement of small unidentifiable inclusions. Dicalcium silicate was often cracked radially and sometimes completely separated by cracking or embayment. The appearance of dicalcium silicate, in plant-cooled clinker, was characteristic of that found in either slowly or quickly cooled clinkers, since plant cooling was intermediate between these two cooling rates.

$\gamma$-Dicalcium silicate, sometimes in considerable amounts, was observed only in slowly cooled clinkers.

\section{DARK INTERSTITAL MATERIAL}

Dark interstitial material consists of three types: rectangular (identified as $3 \mathrm{CaO} \cdot \mathrm{Al}_{2} \mathrm{O}_{3}$ ), dark prismatic interstitial material, and glass.

Tricalcium aluminate $\left(3 \mathrm{CaO} \cdot \mathrm{Al}_{2} \mathrm{O}_{3}\right)$ etched readily in freshly distilled water to dark-gray rectangular masses, occasionally showing square outlines, and was often intimately associated with tetracalcium aluminoferrite. Where present it was distributed throughout the 
clinker structure and sometimes completely occupied the interstices. Roughly square or rectangular shapes were generally observed, but irregular areas appeared sometimes with the trace of one crystal face. There was no evidence of corrosion, twinning, or zoning. The inclusions were dicalcium silicate, tricalcium silicate, and infrequently periclase. Small, scattered, bubble-like inclusions of what may be tetracalcium aluminoferrite occurred.

Tricalcium aluminate was found in slowly cooled clinkers when the $\mathrm{Al}_{2} \mathrm{O}_{3}-\mathrm{Fe}_{2} \mathrm{O}_{3}$ ratio was 1.63 or greater, and not in those of $\mathrm{Al}_{2} \mathrm{O}_{3}-$ $\mathrm{Fe}_{2} \mathrm{O}_{3}$ ratio 0.97 or less. In the group studied, clinkers having $\mathrm{Al}_{2} \mathrm{O}_{3}$ $\mathrm{Fe}_{2} \mathrm{O}_{3}$ ratios between 0.97 and 1.63 were not available.

Only two of the plant-cooled clinkers contained tricalcium aluminate. One of these had an $\mathrm{Al}_{2} \mathrm{O}_{3}-\mathrm{Fe}_{2} \mathrm{O}_{3}$ ratio of 1.89 and a low total alkali content $\left(0.22\right.$ percent). The other had an $\mathrm{Al}_{2} \mathrm{O}_{3}-\mathrm{Fe}_{2} \mathrm{O}_{3}$ ratio of 2.91, the highest in this group, and a total alkali content of 1.00 percent. In this latter case, only a small percentage of the tricalcium aluminate calculated to be present at crystalline equilibrium appeared. In quickly cooled clinkers tricalcium aluminate was not found.

Prismatic dark interstitial material when etched in freshly distilled water appeared as dark-gray, almost black, elongated crystals, frequently with darkened edges. It showed no corrosion, twinning, or zoning. Occasionally the crystals were bordered by ragged, weakly etched dark interstitial material which was identified as glass. Scattered crystals and aggregates appeared throughout the clinker as slender, needle-like, and large, poorly terminated crystals. Frequently they terminated against silicates. In thin sections viewed with transmitted light these crystals were anisotropic with a birefringence of about 0.010 and parallel extinction. Their refractive indices were close to $1.72[6]$.

Prismatic dark interstitial material was much more abundant in plant-cooled than in slowly or quickly cooled clinkers. The quantity of this constituent appeared to be influenced by the rate of cooling. The high glass content of quickly cooled clinkers indicated that rapid cooling prevents crystallization of prismatic dark interstitial material, since this compound is one of the last to crystallize. This phase was more frequent in occurrence and better in development for high $\mathrm{Al}_{2} \mathrm{O}_{3}-\mathrm{Fe}_{2} \mathrm{O}_{3}$ ratio clinkers containing appreciable amounts of $\mathrm{Na}_{2} \mathrm{O}$ and $\mathrm{K}_{2} \mathrm{O}$ either alone or together.

Glass is revealed by etching with 10 percent $\mathrm{KOH}$ [8]. The etchability of glass in other reagents depends on its composition. For instance, glass low in iron is readily etched with water followed by 1 percent $\mathrm{HNO}_{3}$ in alcohol, and glass high in iron is unaffected by this etchant method [10].

The amount of glass varied greatly with different cooling rates. In slowly cooled clinker it occurred infrequently and was seen edging prismatic dark interstitial material. In plant-cooled clinker it occurred as irregular areas, blebs, small veinlets, and frequently as a 
jagged sheath around dark prismatic crystals. Etched, quickly cooled clinkers revealed glass as ragged areas, blebs, and veinlets. Occasionally it had a roughly prismatic form due to prior crystallization of tetracalcium aluminoferrite prisms. When in the form of blebs, it frequently gave the light interstitial material a mottled appearance which made difficult its differentiation from tetracalcium aluminoferrite.

\section{TETRACALCIUM ALUMINOFERRITE}

Tetracalcium aluminoferrite $\left(4 \mathrm{CaO} \cdot \mathrm{Al}_{2} \mathrm{O}_{3} \cdot \mathrm{Fe}_{2} \mathrm{O}_{3}\right)$ was unaffected by most etchants and appeared as light interstitial material intimately associated with dark interstitial material.

In the clinkers investigated, tetracalcium aluminoferrite was the last crystalline phase to appear except in compositions of low $\mathrm{Al}_{2} \mathrm{O}_{3^{-}}$ $\mathrm{Fe}_{2} \mathrm{O}_{3}$ ratios, where the tricalcium aluminate gave every indication of having been the last phase to crystallize.

In slowly cooled clinkers well-developed prisms of tetracalcium aluminoferrite were easily recognized in thin sections. The prism forms varied with the $\mathrm{Al}_{2} \mathrm{O}_{3}-\mathrm{Fe}_{2} \mathrm{O}_{3}$ ratio-large, broad forms predominating in clinkers of low ratio and small needle-like forms in those of high ratio. There was very little change from the amber color and slight pleochroism of the pure compound.

In quickly cooled clinkers tetracalcium aluminoferrite appeared as long, narrow prisms irrespective of the $\mathrm{Al}_{2} \mathrm{O}_{3}-\mathrm{Fe}_{2} \mathrm{O}_{3}$ ratio. It was also frequently found as dentritic growths in clinkers having low $\mathrm{Al}_{2} \mathrm{O}_{3}-\mathrm{Fe}_{2} \mathrm{O}_{3}$ ratios. The large, broad prisms found in slowly cooled clinker were practically absent. In many cases the amber color of this phase was darker and greener while the pleochroism became more pronounced, which may have been due to solid solution with $\mathrm{MgO}$ [11]. Insley and McMurdie [12] pointed out from their studies that there must be less than 1 percent of $\mathrm{MgO}$ in solid solution in tetracalcium aluminoferrite.

\section{COMPARISON OF PHASE COMPOSITIONS AS DETER- MINED MICROSCOPICALLY AND AS CALCULATED FROM ANALYTICAL DATA}

The phases in portland cement clinker are usually calculated from chemical analyses without regard to the rate of clinker cooling. In order to show the inaccuracies which develop from this procedure, comparisons of data obtained from microscopical analyses are made with those obtained from chemical analyses by two methods of calculation.

The percentages by weight of the various phases, calculated from microscopical observations and from chemical analyses, are given in table 2, arranged in order of increasing $\mathrm{Al}_{2} \mathrm{O}_{3}-\mathrm{Fe}_{2} \mathrm{O}_{3}$ ratios for similar 
cooling conditions. The columns designated "Micro" contain results of microscopical analyses. It is necessary to assume in calculating data such as these that each phase is pure and that the effects of zoning and solid solution are negligible. In order to convert volume percentages to weight percentages the following density values [2] were used: $3 \mathrm{CaO} . \mathrm{SiO}_{2}, 3.13 ; \beta-2 \mathrm{CaO} . \mathrm{SiO}_{2}, 3.28 ; 4 \mathrm{CaO} \cdot \mathrm{Al}_{2} \mathrm{O}_{3} \cdot \mathrm{Fe}_{2} \mathrm{O}_{3}$, $3.77 ; \mathrm{CaO}, 3.32 ; \mathrm{MgO}, 3.58$. Since the composition of the glass is variable and uncertain, a density value of 3.00 [2] was used for all dark interstitial material.

TABLE 1.-Chemical analyses of clinkers arranged in order of increasing $\mathrm{Al}_{2} \mathrm{O}_{3} / \mathrm{Fe}_{2} \mathrm{O}_{3}$ for similarly cooled clinkers

[Chemical analyses by C. L. Ford, Research Lab., Portland Cement Assn., Chicago]

\begin{tabular}{|c|c|c|c|c|c|c|c|c|c|c|c|c|c|c|}
\hline$\dot{0}$ & 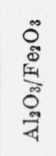 & 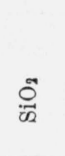 & 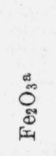 & 脶 & : & 唨 & 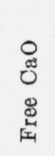 & రీ & 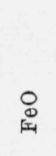 & $\stackrel{\text { }}{\stackrel{\text { H }}{*}}$ & $\begin{array}{l}0 \\
\text { డ్ } \\
\text { Z }\end{array}$ & O్ & 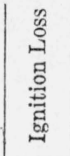 & 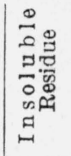 \\
\hline $4{ }_{4}{ }_{4}$ & $\begin{array}{r}0.65 \\
.89 \\
.81\end{array}$ & $\begin{array}{c}\% \\
19.74 \\
19.84 \\
19.51\end{array}$ & $\begin{array}{c}\% \\
7.11 \\
5.33 \\
6.04\end{array}$ & $\begin{array}{c}\% \\
4.61 \\
4.73 \\
4.91\end{array}$ & $\begin{array}{c}\% \\
62.93 \\
58.51 \\
61.24\end{array}$ & $\begin{array}{c}\% \\
4.28 \\
4.24 \\
4.37\end{array}$ & $\begin{array}{c}\% \\
0.30 \\
4.20 \\
1.51\end{array}$ & $\begin{array}{r}\% \\
0.12 \\
.13 \\
.08\end{array}$ & $\begin{array}{c}\% \\
0.00 \\
1.46 \\
1.27\end{array}$ & $\begin{array}{c}\% \\
\text { dN.D. } \\
0.37 \\
\text { N. D. }\end{array}$ & $\begin{array}{r}\% \\
0.41 \\
.53 \\
.37\end{array}$ & $\begin{array}{c}\% \\
0.10 \\
.20 \\
.12\end{array}$ & $\begin{array}{c}\% \\
0.14 \\
.23 \\
.00\end{array}$ & $\begin{array}{l}\% \\
0.11 \\
.39 \\
.21\end{array}$ \\
\hline & $\begin{array}{l}.87 \\
.90 \\
.95\end{array}$ & $\begin{array}{l}22.24 \\
21.98 \\
22.38\end{array}$ & & $\begin{array}{l}\text { 4. } 24 \\
\text { 4. } 54 \\
\text { 4. } 08\end{array}$ & & $\begin{array}{r}0.85 \\
.81 \\
.82\end{array}$ & $\begin{array}{r}0.08 \\
.16 \\
.34\end{array}$ & $\begin{array}{l}.06 \\
.11 \\
.12\end{array}$ & $\begin{array}{r}0.00 \\
.00 \\
.33\end{array}$ & $\begin{array}{l}\text { N. D. } \\
\text { N. }: 3 \text {. }\end{array}$ & $\begin{array}{l}.52 \\
.66 \\
.51\end{array}$ & $\begin{array}{l}.05 \\
.16 \\
.08\end{array}$ & $\begin{array}{l}.14 \\
.18 \\
.11\end{array}$ & $\begin{array}{l}.16 \\
.20 \\
.17\end{array}$ \\
\hline & $\begin{array}{r}.97 \\
1.01 \\
1.12\end{array}$ & $\begin{array}{l}21.27 \\
20.62 \\
20.90\end{array}$ & & & & $\begin{array}{l}1.80 \\
1.85\end{array}$ & $\begin{array}{l}.06 \\
.77 \\
.85\end{array}$ & $\begin{array}{l}.45 \\
.61 \\
.18\end{array}$ & $\begin{array}{l}.05 \\
.15 \\
.63\end{array}$ & $\begin{array}{l}\text { N. D. } \\
\text { N. } .42\end{array}$ & $\begin{array}{l}.27 \\
.33 \\
.24\end{array}$ & $\begin{array}{l}.37 \\
.71 \\
.30\end{array}$ & $\begin{array}{l}.12 \\
.17 \\
.08\end{array}$ & $\begin{array}{l}.20 \\
.15 \\
.26\end{array}$ \\
\hline & $\begin{array}{l}1.63 \\
1.64 \\
1.65\end{array}$ & & & & & & $\begin{array}{l}.07 \\
.61 \\
.79\end{array}$ & $\begin{array}{l}.19 \\
.25 \\
.10\end{array}$ & $\begin{array}{l}.00 \\
.00 \\
.03\end{array}$ & $\begin{array}{l}\text { N. D } \\
\text { N. } .35\end{array}$ & $\begin{array}{l}.15 \\
.17 \\
.10\end{array}$ & & $\begin{array}{l}.17 \\
.25 \\
.19\end{array}$ & $\begin{array}{l}.18 \\
.14 \\
.23\end{array}$ \\
\hline & $\begin{array}{l}1.68 \\
1.68 \\
1.68\end{array}$ & $\begin{array}{l}23.68 \\
23.48 \\
23.69\end{array}$ & & & & & $\begin{array}{r}.18 \\
2.06 \\
1.96\end{array}$ & $\begin{array}{l}.28 \\
.57 \\
.22\end{array}$ & $\begin{array}{l}.00 \\
.00 \\
.00\end{array}$ & $\begin{array}{l}\text { N. D. } \\
\text { N. }{ }^{33} \\
\text { D. }\end{array}$ & $\begin{array}{l}.26 \\
.34 \\
.26\end{array}$ & $\begin{array}{l}.33 \\
.81 \\
.37\end{array}$ & $\begin{array}{l}\text { N. D. } \\
.45 \\
\text { N. D. }\end{array}$ & $\begin{array}{l}\text { N. D. } \\
.20 \\
\text { N. D. }\end{array}$ \\
\hline & $\begin{array}{l}1.88 \\
1.89 \\
1.88\end{array}$ & $\begin{array}{l}21.85 \\
21.67 \\
21.73\end{array}$ & & & & $\begin{array}{l}1.43 \\
1.44 \\
1.48\end{array}$ & $\begin{array}{r}0.00 \\
.36 \\
.47\end{array}$ & $\begin{array}{l}.12 \\
.32 \\
.23\end{array}$ & $\begin{array}{l}.00 \\
.00 \\
.03\end{array}$ & $\begin{array}{l}\text { N. D. } \\
\text { N. }: 26\end{array}$ & $\begin{array}{l}.07 \\
.10 \\
.08\end{array}$ & $\begin{array}{r}\text { trace } \\
.11 \\
.08\end{array}$ & $\begin{array}{l}.17 \\
.17 \\
.12\end{array}$ & $\begin{array}{l}.21 \\
.18 \\
.23\end{array}$ \\
\hline$S_{P}$ & $\begin{array}{l}1.88 \\
2.40 \\
2.60\end{array}$ & $\begin{array}{l}22.72 \\
22.35 \\
22.48\end{array}$ & $\begin{array}{l}2.71 \\
2.24 \\
1.98\end{array}$ & & & $\begin{array}{l}\text { 3. } 99 \\
\text { 4. } 03\end{array}$ & $\begin{array}{l}.16 \\
.38 \\
.37\end{array}$ & $\begin{array}{l}.03 \\
.11 \\
.04\end{array}$ & $\begin{array}{l}.00 \\
.39 \\
.66\end{array}$ & $\begin{array}{l}\text { N. D. } \\
\text { N. } .50\end{array}$ & $\begin{array}{l}.35 \\
.63 \\
.47\end{array}$ & $\begin{array}{r}\text { trace } \\
.20 \\
.10\end{array}$ & $\begin{array}{l}.14 \\
.25 \\
.07\end{array}$ & $\begin{array}{l}.18 \\
.22 \\
.28\end{array}$ \\
\hline$S_{-. .}$ & $\begin{array}{l}2.51 \\
2.51 \\
2.84\end{array}$ & $\begin{array}{l}22.80 \\
22.68 \\
22.79\end{array}$ & $\begin{array}{l}2.23 \\
2.22 \\
1.97\end{array}$ & $\begin{array}{l}5.59 \\
5.56 \\
5.59\end{array}$ & & $\begin{array}{l}4.15 \\
4.15\end{array}$ & $\begin{array}{l}.10 \\
.80 \\
.85\end{array}$ & $\begin{array}{l}.13 \\
.28 \\
.11\end{array}$ & $\begin{array}{l}.00 \\
.00 \\
.23\end{array}$ & $\begin{array}{l}\text { N. D. } \\
\text { N. }{ }^{30}\end{array}$ & $\begin{array}{l}.32 \\
.38 \\
.33\end{array}$ & $\begin{array}{l}.22 \\
.54 \\
.29\end{array}$ & $\begin{array}{l}\text { N. D. } \\
: 26 \\
\text { N. D. }\end{array}$ & $\begin{array}{l}\text { N. D. } \\
\text { N. } .30 \\
\text { D. }\end{array}$ \\
\hline $1 \mathrm{~S}$ & $\begin{array}{l}2.70 \\
2.71 \\
3.00\end{array}$ & $\begin{array}{l}21.70 \\
21.46 \\
21.75\end{array}$ & $\begin{array}{l}2.21 \\
2.22 \\
1.90\end{array}$ & $\begin{array}{l}5.97 \\
6.03 \\
5.71\end{array}$ & $\begin{array}{l}64.64 \\
63.05 \\
63.58\end{array}$ & $\begin{array}{l}\text { 3. } 08 \\
\text { 3. } 01 \\
\text { 3. } 08\end{array}$ & $\begin{array}{r}.38 \\
1.21 \\
1.53\end{array}$ & $\begin{array}{l}.50 \\
.68 \\
.49\end{array}$ & $\begin{array}{l}.00 \\
.00 \\
.30\end{array}$ & $\begin{array}{l}\text { N. D. } \\
\dot{\text { N. }} \cdot \mathbf{3 1}\end{array}$ & $\begin{array}{l}.10 \\
.10\end{array}$ & $\begin{array}{r}.97 \\
1.58 \\
1.11\end{array}$ & $\begin{array}{l}.20 \\
.36 \\
.19\end{array}$ & $\begin{array}{l}.25 \\
.24 \\
.19\end{array}$ \\
\hline $\begin{array}{l}\gamma_{S} S \\
\gamma_{P}\end{array}$ & $\begin{array}{l}2.82 \\
2.91 \\
3.47\end{array}$ & $\begin{array}{l}21.40 \\
21.12 \\
21.20\end{array}$ & $\begin{array}{l}2.35 \\
2.35 \\
1.96\end{array}$ & $\begin{array}{l}6.64 \\
6.85 \\
6.80\end{array}$ & $\begin{array}{l}64.73 \\
63.81 \\
64.10\end{array}$ & $\begin{array}{l}3.42 \\
3.48\end{array}$ & $\begin{array}{r}0.02 \\
.41 \\
.55\end{array}$ & $\begin{array}{l}.29 \\
.36 \\
.22\end{array}$ & $\begin{array}{l}.00 \\
.03 \\
.36\end{array}$ & $\begin{array}{l}\text { N. D } \\
\text { N. D. }\end{array}$ & $\begin{array}{l}.34 \\
.42 \\
.32\end{array}$ & $\begin{array}{r}0.28 \\
.58 \\
.38\end{array}$ & $\begin{array}{l}.14 \\
.15 \\
.11\end{array}$ & $\begin{array}{r}.15 \\
.23 \\
.21\end{array}$ \\
\hline
\end{tabular}

a Corrected for $\mathrm{FeO}$.

b Corrected for $\mathrm{TiO}_{2}$

- Corrected for free $\mathrm{CaO}$.

d N. D. = Not determined. 
[Percentages by weight]

\begin{tabular}{|c|c|c|c|c|c|c|c|c|c|c|c|c|c|c|c|c|c|c|c|c|c|c|c|c|c|}
\hline No. ${ }^{8}$ & 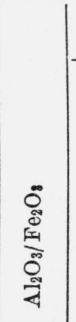 & \multicolumn{3}{|c|}{$3 \mathrm{CaO} . \mathrm{SiO}_{2}$} & \multicolumn{3}{|c|}{$2 \mathrm{CaO} . \mathrm{SiO}_{2}$} & \multicolumn{3}{|c|}{$3 \mathrm{CaO} . \mathrm{Al}_{2} \mathrm{O}_{3}$} & \multicolumn{4}{|c|}{$4 \mathrm{CaO} \cdot \mathrm{Al}_{2} \mathrm{O}_{3} \cdot \mathrm{Fe}_{2} \mathrm{O}_{3}$} & \multicolumn{3}{|c|}{$\mathrm{MgO}$} & \multicolumn{2}{|c|}{$\mathrm{CaO}$} & \multicolumn{3}{|c|}{ Glass } & 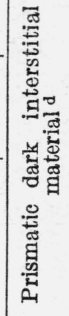 & 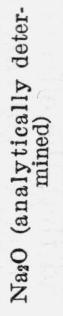 & 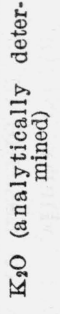 \\
\hline & $\begin{array}{l}.87 \\
.90 \\
.95\end{array}$ & $\begin{array}{l}66 \\
64 \\
67\end{array}$ & $\begin{array}{l}66 \\
64 \\
67\end{array}$ & $\begin{array}{l}67 \\
65 \\
73\end{array}$ & $\begin{array}{l}14 \\
15 \\
14\end{array}$ & $\begin{array}{l}13 \\
13 \\
12\end{array}$ & $\begin{array}{l}13 \\
14 \\
10\end{array}$ & $\begin{array}{l}3 \\
4 \\
4\end{array}$ & $\begin{array}{l}2 \\
2 \\
1\end{array}$ & $\begin{array}{l}0 \\
0 \\
0\end{array}$ & $\begin{array}{l}15 \\
15 \\
13\end{array}$ & $\begin{array}{l}14 \\
12 \\
10\end{array}$ & $\begin{array}{l}15 \\
15 \\
10\end{array}$ & $\begin{array}{l}15 \\
17 \\
14\end{array}$ & $\begin{array}{r}0.9 \\
.8 \\
.8\end{array}$ & $\begin{array}{r}0.7 \\
.4 \\
.4\end{array}$ & $\begin{array}{r}0.8 \\
.6 \\
.2\end{array}$ & $\begin{array}{r}0.1 \\
.2 \\
.3\end{array}$ & $\begin{array}{r}0.1 \\
.1 \\
.1\end{array}$ & $\begin{array}{l}2 \\
6 \\
7\end{array}$ & $\begin{array}{l}1 \\
4 \\
7\end{array}$ & $\begin{array}{l}1 \\
3 \\
3\end{array}$ & $\begin{array}{l}3 \\
0 \\
0\end{array}$ & $\begin{array}{l}.52 \\
.66 \\
.51\end{array}$ & .08 \\
\hline & $\begin{array}{l}.97 \\
1.01 \\
1.12\end{array}$ & $\begin{array}{l}63 \\
56 \\
58\end{array}$ & $\begin{array}{l}62 \\
56 \\
58\end{array}$ & $\begin{array}{l}64 \\
57 \\
64\end{array}$ & $\begin{array}{l}14 \\
17 \\
16\end{array}$ & $\begin{array}{l}14 \\
16 \\
13\end{array}$ & $\begin{array}{l}15 \\
11 \\
13\end{array}$ & $\begin{array}{l}5 \\
5 \\
6\end{array}$ & $\begin{array}{l}4 \\
4 \\
0.2\end{array}$ & $\begin{array}{l}0 \\
0 \\
0\end{array}$ & $\begin{array}{l}16 \\
16 \\
15\end{array}$ & $\begin{array}{r}15 \\
14 \\
6\end{array}$ & $\begin{array}{l}17 \\
20 \\
11\end{array}$ & $\begin{array}{l}17 \\
26 \\
19\end{array}$ & $\begin{array}{l}1.7 \\
1.8 \\
1.9\end{array}$ & $\begin{array}{l}1.6 \\
1.5 \\
0.7\end{array}$ & $\begin{array}{l}1.3 \\
2.2 \\
0.7\end{array}$ & $\begin{array}{l}.1 \\
.8 \\
.9\end{array}$ & $\begin{array}{l}0.1 \\
1.0 \\
1.2\end{array}$ & $\begin{array}{r}1 \\
5 \\
20\end{array}$ & $\begin{array}{l}0 \\
6 \\
8\end{array}$ & $\begin{array}{l}0 \\
0 \\
3\end{array}$ & $\begin{array}{l}3 \\
3 \\
0 \\
0\end{array}$ & $\begin{array}{l}.27 \\
.33 \\
.24\end{array}$ & $\begin{array}{l}.37 \\
.71 \\
.30\end{array}$ \\
\hline${ }_{6 P}^{6 S}$ & $\begin{array}{l}1.88 \\
1.89 \\
1.88\end{array}$ & $\begin{array}{l}63 \\
61 \\
61\end{array}$ & $\begin{array}{l}63 \\
61 \\
61\end{array}$ & $\begin{array}{l}71 \\
62 \\
70\end{array}$ & $\begin{array}{l}15 \\
16 \\
17\end{array}$ & $\begin{array}{l}14 \\
16 \\
14\end{array}$ & $\begin{array}{r}9 \\
16 \\
10\end{array}$ & $\begin{array}{l}10 \\
11 \\
10\end{array}$ & $\begin{array}{r}9 \\
10 \\
6\end{array}$ & $\begin{array}{l}6 \\
7 \\
0\end{array}$ & $\begin{array}{r}9 \\
10 \\
10\end{array}$ & $\begin{array}{l}8 \\
9 \\
2\end{array}$ & $\begin{array}{r}11 \\
10 \\
2\end{array}$ & $\begin{array}{l}11 \\
10 \\
10\end{array}$ & $\begin{array}{l}1.4 \\
1.4 \\
1.5\end{array}$ & $\begin{array}{l}1.2 \\
1.3 \\
0.6\end{array}$ & $\begin{array}{l}.4 \\
: 9 \\
.2\end{array}$ & $\begin{array}{l}0 \\
0.4 \\
.5\end{array}$ & $\begin{array}{l}0.1 \\
.7 \\
1.0\end{array}$ & $\begin{array}{r}4 \\
2 \\
15\end{array}$ & $\begin{array}{r}2 \\
3 \\
17\end{array}$ & $\begin{array}{l}2 \\
3 \\
9\end{array}$ & $\begin{array}{l}0 \\
0.6\end{array}$ & $\begin{array}{l}.07 \\
.10 \\
.08\end{array}$ & $\begin{array}{r}\text { trace } \\
.11 \\
.08\end{array}$ \\
\hline $\begin{array}{l}B S \\
B P \\
B Q\end{array}$ & \begin{tabular}{l|l}
1.88 \\
2.40 \\
2.60
\end{tabular} & $\begin{array}{l}50 \\
49 \\
52\end{array}$ & $\begin{array}{l}51 \\
49 \\
56\end{array}$ & $\begin{array}{l}54 \\
59 \\
66\end{array}$ & $\begin{array}{l}27 \\
27 \\
26\end{array}$ & $\begin{array}{l}27 \\
25 \\
19\end{array}$ & $\begin{array}{l}25 \\
17 \\
17\end{array}$ & $\begin{array}{r}9 \\
10 \\
10\end{array}$ & $\begin{array}{l}9 \\
7 \\
0\end{array}$ & $\begin{array}{l}7 \\
0 \\
0\end{array}$ & $\begin{array}{l}8 \\
7 \\
6\end{array}$ & $\left|\begin{array}{l}8 \\
1 \\
0\end{array}\right|$ & $\left|\begin{array}{r}4 \\
1 \\
\text { trace }\end{array}\right|$ & $\begin{array}{l}4 \\
2 \\
1\end{array}$ & $\begin{array}{l}4.1 \\
4.0 \\
4.0\end{array}$ & $\begin{array}{l}4.1 \\
3.3 \\
3.0\end{array}$ & $\begin{array}{l}4.0 \\
4.1 \\
2.1\end{array}$ & $\begin{array}{l}.2 \\
.4 \\
.4\end{array}$ & $\begin{array}{r}0.2 \\
.4 \\
.1\end{array}$ & $\begin{array}{r}0 \\
12 \\
18\end{array}$ & $\begin{array}{r}2 \\
8 \\
11\end{array}$ & $\begin{array}{r}2 \\
7 \\
10\end{array}$ & $\begin{array}{r}4 \\
10 \\
5\end{array}$ & $\begin{array}{l}.35 \\
.63 \\
.47\end{array}$ & $\begin{array}{r}\text { trace } \\
.20 \\
.10\end{array}$ \\
\hline
\end{tabular}




\begin{tabular}{|c|c|c|c|c|c|c|c|c|c|c|c|c|c|c|c|c|c|c|c|c|c|c|c|c|c|}
\hline${ }_{9 P}{ }_{9 Q} Q_{-}$ & $\begin{array}{l}2.51 \\
2.51 \\
2.84\end{array}$ & $\begin{array}{l}45 \\
42 \\
43\end{array}$ & $\begin{array}{l}46 \\
43 \\
45\end{array}$ & $\begin{array}{l}45 \\
49 \\
52\end{array}$ & $\begin{array}{l}31 \\
33 \\
33\end{array}$ & $\begin{array}{l}31 \\
31 \\
28\end{array}$ & $\begin{array}{l}37 \\
34 \\
32\end{array}$ & $\begin{array}{l}11 \\
11 \\
12\end{array}$ & $\begin{array}{r}11 \\
8 \\
5\end{array}$ & $\begin{array}{r}10 \\
0 \\
0\end{array}$ & $\begin{array}{l}7 \\
7 \\
6\end{array}$ & $\begin{array}{l}7 \\
2 \\
0\end{array}$ & $\begin{array}{r}5 \\
6 \\
\text { trace }\end{array}$ & $\begin{array}{l}5 \\
6 \\
5\end{array}$ & $\begin{array}{l}4.2 \\
4.2 \\
4.2\end{array}$ & $\begin{array}{l}\text { 4. } 2 \\
\text { 3. } 6 \\
3.2\end{array}$ & $\begin{array}{l}2.8 \\
2.5 \\
2.5\end{array}$ & $\begin{array}{l}.1 \\
.8 \\
.9\end{array}$ & $\begin{array}{l}.2 \\
.7 \\
.8\end{array}$ & $\begin{array}{r}0 \\
9 \\
16\end{array}$ & $\begin{array}{r}0 \\
7 \\
13\end{array}$ & $\begin{array}{l}0 \\
7 \\
8\end{array}$ & $\begin{array}{l}0 \\
2 \\
0\end{array}$ & $\begin{array}{l}.32 \\
.38 \\
.33\end{array}$ & $\begin{array}{l}.22 \\
.54 \\
.29\end{array}$ \\
\hline $\begin{array}{l}1 S_{1} \\
1 Q \\
1 Q\end{array}$ & $\begin{array}{l}2.70 \\
2.71 \\
\text { 3. } 00\end{array}$ & $\begin{array}{l}55 \\
50 \\
52\end{array}$ & $\begin{array}{l}55 \\
50 \\
52\end{array}$ & $\begin{array}{l}45 \\
55 \\
58\end{array}$ & $\begin{array}{l}21 \\
24 \\
23\end{array}$ & $\begin{array}{l}21 \\
24 \\
21\end{array}$ & $\begin{array}{l}33 \\
24 \\
19\end{array}$ & $\begin{array}{l}12 \\
12 \\
12\end{array}$ & $\begin{array}{r}12 \\
12 \\
9\end{array}$ & $\begin{array}{r}12 \\
\text { trace } \\
0\end{array}$ & $\begin{array}{l}7 \\
7 \\
6\end{array}$ & 7 & $\begin{array}{l}7 \\
4 \\
6\end{array}$ & $\begin{array}{l}7 \\
4 \\
6\end{array}$ & $\begin{array}{l}3.1 \\
3.0 \\
3.1\end{array}$ & $\begin{array}{l}3.1 \\
2.9 \\
2.5\end{array}$ & $\begin{array}{l}2.4 \\
2.5 \\
1.1\end{array}$ & $\begin{array}{r}.4 \\
1.2 \\
1.5\end{array}$ & $\begin{array}{l}.4 \\
.9 \\
.3\end{array}$ & $\begin{array}{r}0 \\
3 \\
10\end{array}$ & $\begin{array}{r}0 \\
1 \\
14\end{array}$ & $\begin{array}{r}0 \\
1 \\
14\end{array}$ & $\begin{array}{r}0 \\
13 \\
2\end{array}$ & $\begin{array}{l}.1 \\
.1 \\
.1\end{array}$ & $\begin{array}{l}.97 \\
1.58 \\
1.11\end{array}$ \\
\hline 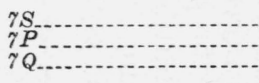 & $\begin{array}{l}2.82 \\
2.91 \\
3.47\end{array}$ & $\begin{array}{l}53 \\
50 \\
51\end{array}$ & $\begin{array}{l}53 \\
50 \\
58\end{array}$ & $\begin{array}{l}55 \\
46 \\
61\end{array}$ & $\begin{array}{l}22 \\
23 \\
22\end{array}$ & $\begin{array}{l}21 \\
22 \\
12\end{array}$ & $\begin{array}{l}24 \\
27 \\
18\end{array}$ & $\begin{array}{l}14 \\
14 \\
15\end{array}$ & $\begin{array}{r}13 \\
11 \\
0\end{array}$ & $\begin{array}{r}12 \\
2 \\
0\end{array}$ & $\begin{array}{l}7 \\
7 \\
6\end{array}$ & 0 & $\begin{array}{r}5 \\
2 \\
\text { trace }\end{array}$ & $\begin{array}{l}5 \\
8 \\
4\end{array}$ & $\begin{array}{l}3.4 \\
3.4 \\
3.5\end{array}$ & $\begin{array}{l}3.3 \\
2.9 \\
1.9\end{array}$ & $\begin{array}{l}2.8 \\
4.1 \\
1.7\end{array}$ & $\begin{array}{l}0 \\
.4 \\
.6\end{array}$ & $\begin{array}{r}.1 \\
.5 \\
2.0\end{array}$ & $\begin{array}{r}2 \\
9 \\
26\end{array}$ & $\begin{array}{r}2 \\
12 \\
18\end{array}$ & $\begin{array}{r}2 \\
6 \\
14\end{array}$ & $\begin{array}{l}0.3 \\
7 \\
0.2\end{array}$ & $\begin{array}{l}.34 \\
.42 \\
.32\end{array}$ & $\begin{array}{r}0.28 \\
.58 \\
.38\end{array}$ \\
\hline
\end{tabular}

a $S=$ Slowly cooled clinker; $P=$ plant clinker; $Q=$ quickly cooled clinker.

Calculated assuming complete equilibrium crystallization with allowance made for free $\mathrm{CaO}, \mathrm{TiO}_{2}$, and $\mathrm{FeO}$.

作

- Revised values based on heats of solution of glasses on $\mathrm{T}_{2} \mathrm{E}, \mathrm{T}_{2} \mathrm{~W}$ [14] boundary curves and the $1,400^{\circ}$ and $1,450^{\circ}$ isotherms. 
The corrected chemical analyses of the clinkers studied are given in table 1 . The values in this tabulation were used in calculating the results in table 2 . It will be noted that in a given clinker the $\mathrm{Al}_{2} \mathrm{O}_{3}-\mathrm{Fe}_{2} \mathrm{O}_{3}$ ratios generally are lower in the slowly than in the quickly cooled clinkers. This is because the constituents in this ratio were corrected for ferrous oxide. Under conditions of crystalline equilibrium the calculated $3 \mathrm{CaO} \cdot \mathrm{Al}_{2} \mathrm{O}_{3}$ should increase and the calculated $4 \mathrm{CaO} \cdot \mathrm{Al}_{2} \mathrm{O}_{3} \cdot \mathrm{Fe}_{2} \mathrm{O}_{3}$ decrease with increased $\mathrm{Al}_{2} \mathrm{O}_{3}-\mathrm{Fe}_{2} \mathrm{O}_{3}$ ratios. An examination of table 2 shows this to be the case.

But under those conditions of crystallization where significant amounts of glass persist after the cooling of the clinker, there should be less of the phases, $3 \mathrm{CaO} \cdot \mathrm{Al}_{2} \mathrm{O}_{3}$ and $4 \mathrm{CaO} \cdot \mathrm{Al}_{2} \mathrm{O}_{3} \cdot \mathrm{Fe}_{2} \mathrm{O}_{3}$, since part of these constituents would remain in the glass. The effects should be more pronounced in the more quickly cooled clinkers. Methods of calculation which take account of this residual glass should show this effect. The values obtained microscopically decrease with increased cooling rate, which indicates that the rate of cooling is a significant factor to be considered in calculating clinker phases.

The columns designated "equilibrium" and "normal" in table 2 contain results derived from chemical analyses by two methods of calculation. Under the subheading "normal" are given the results by the method of L. A. Dahl [13], which are an extension of the derivations published by Lea and Parker [14]. Included in the derivations of Dahl are equations for calculating the phase composition under the assumption that the clinker is at equilibrium down to a temperature when the liquid remaining is just equal to the glass content as determined by the heat-of-solution method. Thus glass as used in the resulting computations is an approximated value, and the remaining crystalline phases are calculated by the equations given. The other method is based upon an assumption of complete equilibrium during the cooling of the clinker [15].

The magnesia is included in the Dahl calculations under the assumption that $\mathrm{MgO}$ dissolves in the liquid up to 6 percent [16] and that any $\mathrm{MgO}$ remaining crystallizes as periclase. Further modifications will be required when the part played by the alkalies and other minor oxides is known. However, the method represents the best method of calculation from chemical analysis available at this time.

In making the calculations, allowance was made for free $\mathrm{CaO}, \mathrm{FeO}$, and $\mathrm{TiO}_{2}$. Free $\mathrm{CaO}$ was subtracted from the total $\mathrm{CaO} ; \mathrm{TiO}_{2}$ from $\mathrm{Al}_{2} \mathrm{O}_{3}$; and $\mathrm{FeO}$ as $\mathrm{Fe}_{2} \mathrm{O}_{3}$ was subtracted from $\mathrm{Fe}_{2} \mathrm{O}_{3}$ to give the amounts of $\mathrm{CaO}, \mathrm{Al}_{2} \mathrm{O}_{3}$, and $\mathrm{Fe}_{2} \mathrm{O}_{3}$, respectively, which were assumed to participate in the reactions and were, therefore, used in the calculations. The values given for approximated glass content are revised values based on heats of solution of glass on the $\mathrm{T}_{2} \mathrm{~W}, \mathrm{~T}_{2} \mathrm{E}$ boundary curves ${ }^{4}$ and the $1,400^{\circ}$ and $1,450^{\circ} \mathrm{C}$ isotherms [13].

Comparisons between clinker minerals variously determined are also made graphically in figures $5,6(A$ and $B), 7(A$ and $B)$, and 8 $(A$ and $B)$. In each of these figures the clinkers are arranged according to increasing microscopically determined content of the particular component being studied. Each figure shows, in addition to the microscopical values, those values calculated assuming "normal" crystallization and those calculated assuming crystalline equilibrium.

\footnotetext{
- Boundary curves in the quaternary system as discussed by Lea and Parker. See reference [14].
} 
The following abbreviations are used in the figures: $\mathrm{C}_{2} \mathrm{~S}=2 \mathrm{CaO} \cdot \mathrm{SiO}_{2}$, $\mathrm{C}_{3} \mathrm{~S}=3 \mathrm{CaO} . \mathrm{SiO}_{2}, \mathrm{C}_{3} \mathrm{~A}=3 \mathrm{CaO} \cdot \mathrm{Al}_{2} \mathrm{O}_{3}, \mathrm{C}_{4} \mathrm{AF}=4 \mathrm{CaO} \cdot \mathrm{Al}_{2} \mathrm{O}_{3} \cdot \mathrm{Fe}_{2} \mathrm{O}_{3}$.

In figure $6(B)$ the amount of total alkalies is compared with increasing prismatic dark interstitial material as determined microscopically because, although the composition of the prismatic dark interstitial material is unknown, there is reason to suspect a connection with the total alkali content of the clinker. Under the assumption of crystalline equilibrium there can be no residual glass; hence glass is not plotted for this condition in figure $7(B)$.

In general, the figures show a better agreement between microscopically determined values and those calculated assuming "normal" crystallization than between microscopically determined values and
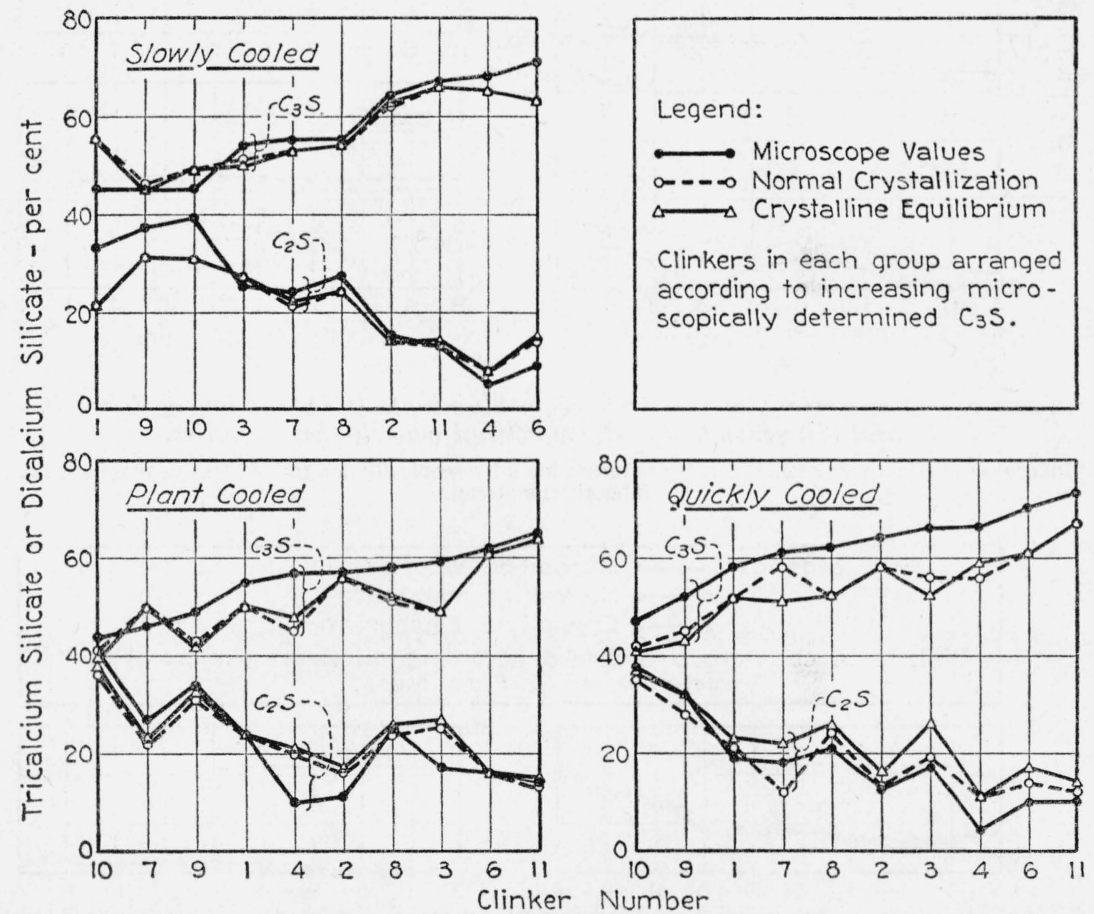

FIGURE 5.-Comparisons of tricalcium silicate $\left(\mathrm{C}_{3} \mathrm{~S}\right)$ and dicalcium silicate $\left(\mathrm{C}_{2} \mathrm{~S}\right)$ variously determined.

those calculated assuming complete equilibrium. For each constituent, agreement is better for slowly cooled clinkers than for either plant-cooled or quickly cooled clinkers. In general, plant-cooled clinkers show better agreement with the calculated phases than quickly cooled clinkers.

Inspection of the tables and figure 5 shows that for the slowly cooled clinkers the values for $3 \mathrm{CaO} . \mathrm{SiO}_{2}$ and $2 \mathrm{CaO} . \mathrm{SiO}_{2}$ calculated from chemical analyses agree reasonably well with those derived from microscopical observations. There is a tendency even here for the calculated values for $3 \mathrm{CaO} . \mathrm{SiO}_{2}$ to be somewhat low and those for $2 \mathrm{CaO} . \mathrm{SiO}_{2}$ to be somewhat high. 

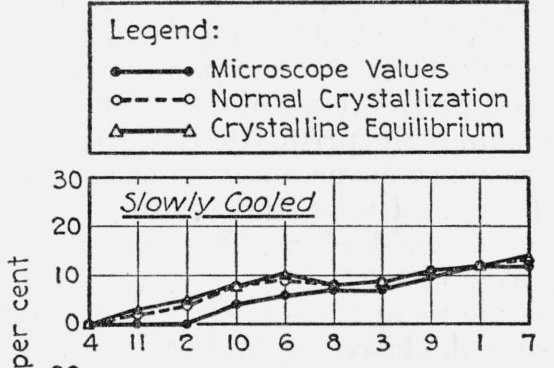

○.
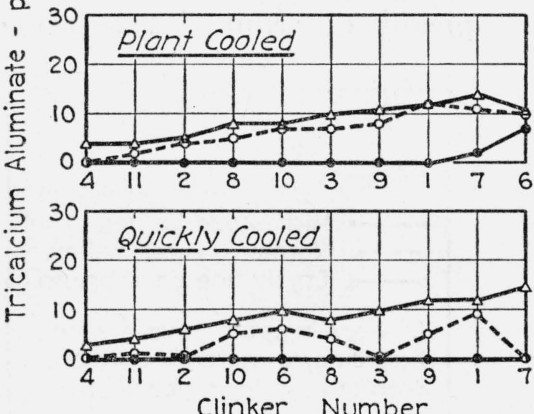

Clinker
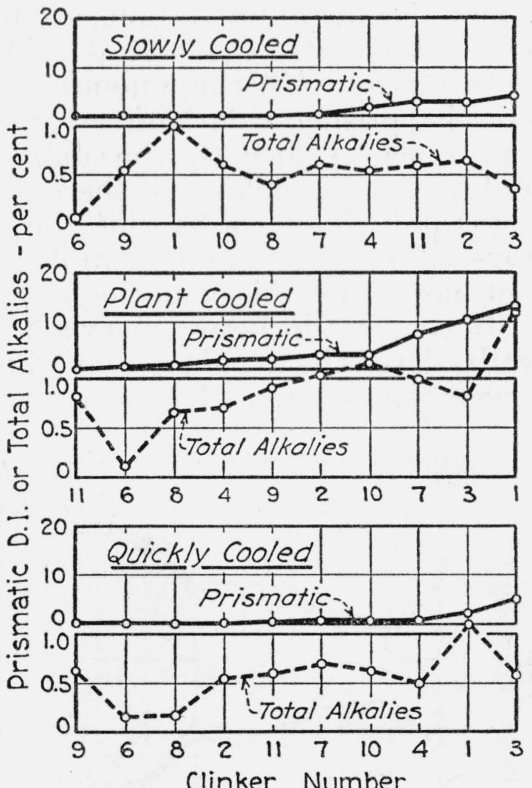

$\mathrm{B}$

Figure 6.-Comparisons of $(A)$ tricalcium aluminate $\left(\mathrm{C}_{3} \mathrm{~A}\right)$ variously determined, and $(B)$ prismatic dark interstitial material and alkalies.

Clinkers in each group arranged according to increasing microscopically determined $\mathrm{C}_{\mathbf{3}} \mathrm{A}$ or prismatic dark interstitial material.

\begin{tabular}{|c|c|}
\hline Legend: & $\begin{array}{l}\longrightarrow \text { Microscope Values } \\
\sim-\infty \text { Normal Crystallization } \\
\leftrightarrow \text { Crystalline Equilibrium }\end{array}$ \\
\hline Clinkers in each 9 & $\begin{array}{l}\text { oup arranged according to increasing microscopically } \\
\text { determined } \mathrm{C}_{4} \mathrm{AF} \text { or Glass. }\end{array}$ \\
\hline
\end{tabular}
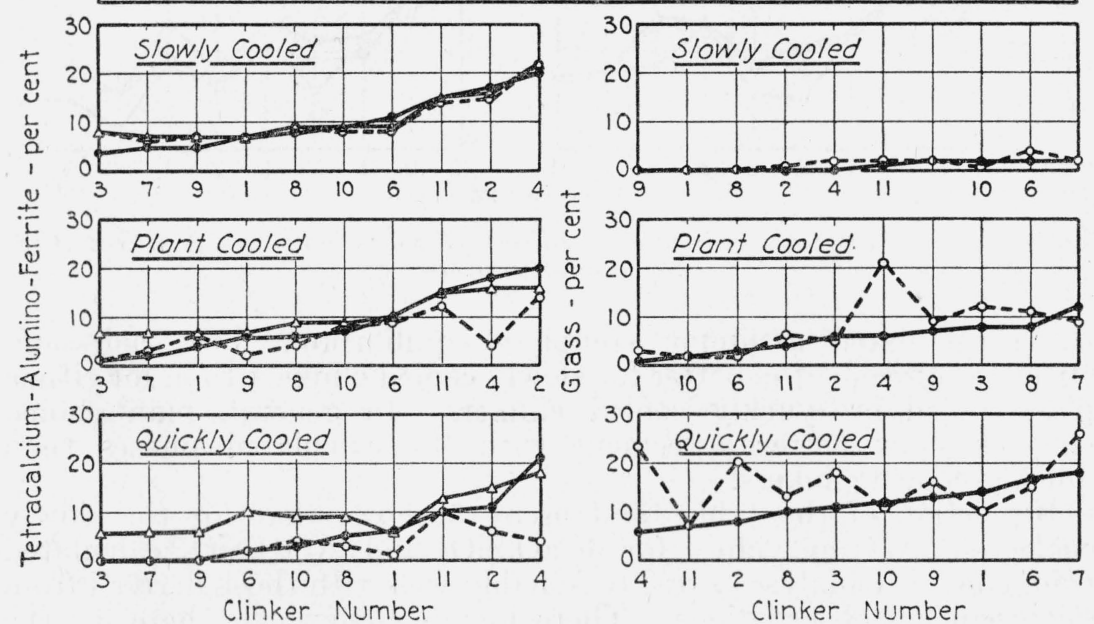

A

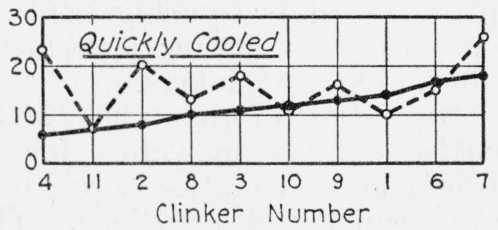

$\mathrm{B}$

FIGURE 7.-Comparisons of $(A)$ tetracalcium aluminoferrite $\left(\mathrm{C}_{4} \mathrm{AF}\right)$ variously determined, and $(B)$ glass variously determined. 
This suggests that crystalline equilibrium has not been completely attained, or that the phase identified as $3 \mathrm{CaO} . \mathrm{SiO}_{2}$ has been augmented by solid solution. In the plant-cooled and the quickly cooled clinkers the above noted tendency becomes marked and definite.

The two dark interstitial crystalline phases etched by water are divided into rectangular and prismatic phases. The rectangular phase agrees optically with tricalcium aluminate while the prismatic phase does not. W. C. Taylor, of this laboratory, suggests that this prismatic phase may be another crystalline form of tricalcium aluminate. It is noteworthy in this connection that the sum of these two microscopically determined crystalline phases, rectangular and pris-

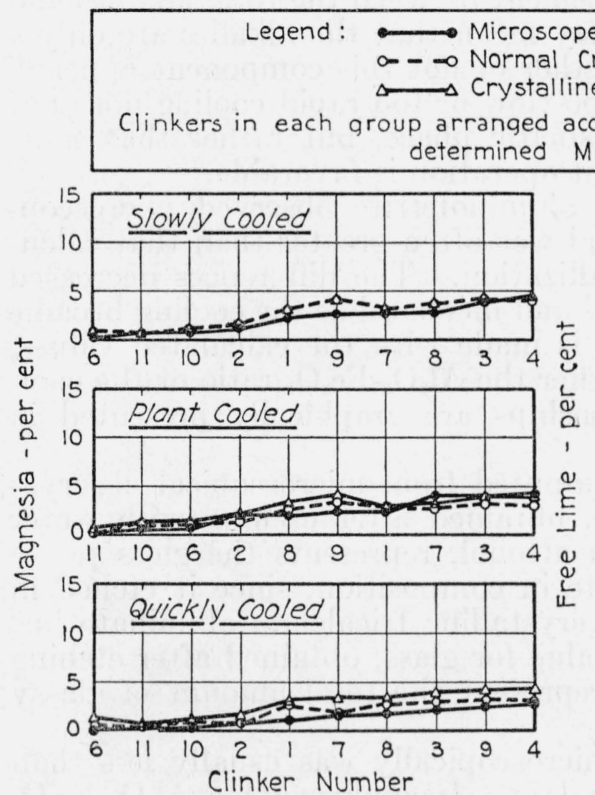

A

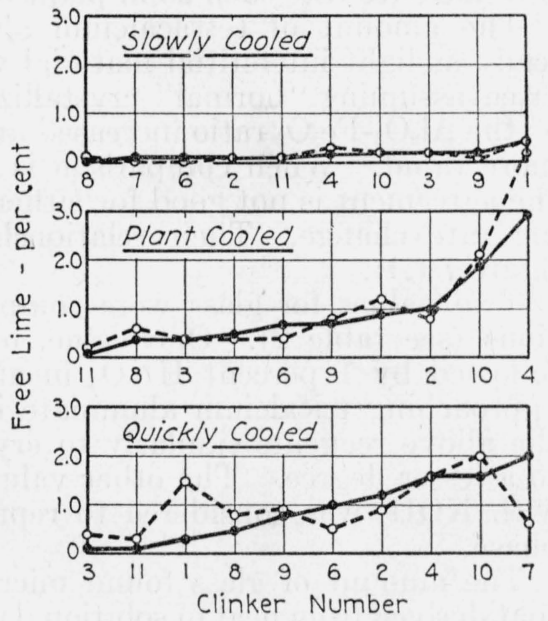

$\mathrm{B}$

FIGURE 8.-Comparisons of $(A)$ magnesia $(\mathrm{MgO})$ variously determined, and $(B)$ free lime $(\mathrm{CaO})$ variously determined.

matic, approaches the values for total tricalcium aluminate as figured by the "normal" crystallization method of calculation. It is Taylor's premise that the rate of cooling and the presence of alkalies have an effect on the type of crystalline dark interstitial material found.

Tricalcium aluminate as a rectangular crystalline phase was found microscopically in all slowly cooled clinkers with $\mathrm{Al}_{2} \mathrm{O}_{3}-\mathrm{Fe}_{2} \mathrm{O}_{3}$ ratios of 1.63 or greater. Lack of clinkers with $\mathrm{Al}_{2} \mathrm{O}_{3}-\mathrm{Fe}_{2} \mathrm{O}_{3}$ ratios between 0.97 and 1.63 prevents a more definite statement as to the minimum $\mathrm{Al}_{2} \mathrm{O}_{3}-\mathrm{Fe}_{2} \mathrm{O}_{3}$ ratio at which this phase appears microscopically in slowly cooled clinkers. Tricalcium aluminate (rectangular) was not found in quickly cooled clinkers and was found in only two plantcooled clinkers $(6 P$ and $7 P)$. In one plant clinker, $6 P$, the total alkalies are very low, 0.21 percent; and in the other instance, $7 P$, where a small percentage of tricalcium aluminate was found, the $\mathrm{Al}_{2} \mathrm{O}_{3}-\mathrm{Fe}_{2} \mathrm{O}_{3}$ ratio is the highest, 2.91, of all plant-cooled clinkers 
studied. The observed amount of rectangular tricalcium aluminate was always less than that calculated. The negative difference increased as the $\mathrm{Al}_{2} \mathrm{O}_{3}-\mathrm{Fe}_{2} \mathrm{O}_{3}$ ratio increased and as the cooling became more rapid. Figure $6(A)$ clearly demonstrates that the formation of rectangular tricalcium aluminate, assuming that the composition was favorable, is dependent upon the rate of cooling.

Since the chemical composition of the prismatic dark interstitial phase is not known, no comparisons were made with the determined microscopical values. Untreated plant clinkers were highest in this prismatic phase and quickly cooled clinkers lowest, possibly because of the higher glass content of the latter. Figure $6(B)$ points out distinctly that there is little agreement between the total alkalies and the prismatic material. This may mean that the alkalies are only a minor factor in determining whether or not this component appears. It seems from the curves that too slow or too rapid cooling does not favor the formation of the prismatic phase, but rather that some intermediate rate such as in plant operation is favorable.

The amount of tetracalcium aluminoferrite observed microscopically as light interstitial material was often greater than that calculated assuming "normal" crystallization. The differences decreased as the $\mathrm{Al}_{2} \mathrm{O}_{3}-\mathrm{Fe}_{2} \mathrm{O}_{3}$ ratio increased and increased as the cooling became more rapid. When comparison is made with the calculated values, the agreement is not good for either the $\mathrm{Al}_{2} \mathrm{O}_{3}-\mathrm{Fe}_{2} \mathrm{O}_{3}$ ratio or the cooling rate change. Their relationships are graphically presented in figure $7(A)$.

Two values for glass were computed from microscopical observations (see table 2). One value, obtained after etching with water followed by 1 percent $\mathrm{HNO}_{3}$ in alcohol, represents the glass phase approaching tricalcium aluminate in composition, since it etched in the above reagents similarly to crystalline tricalcium aluminate but to a lesser degree. The other value for glass, obtained after etching with $\mathrm{KOH}$, was considered to represent the total amount of glassy phase.

The amount of glass found microscopically was usually less than that derived from heat of solution data. In clinkers of low $\mathrm{Al}_{2} \mathrm{O}_{3}-\mathrm{Fe}_{2} \mathrm{O}_{3}$ ratio it was considerably less. In many cases, however, there was fairly good agreement between the amounts of glass found microscopically after etching with $\mathrm{KOH}$ and those calculated from heat of solution data.

It is interesting to note in figure $7(B)$, that as the glass content approached a maximum, as determined by the heat of solution method, the microscope failed to find equivalent amounts. Glass was difficult to determine microscopically, but it is not believed that the large differences shown in some instances were due to failure in identification. There may be some factors not yet recognized, perhaps in both procedures, which when eliminated will permit closer agreement.

Agreement between the values for free lime and magnesia determined microscopically and calculated (analytical values for free lime) was usually good. No consistent variation was observed between these values either with the heat treatment or with the $\mathrm{Al}_{2} \mathrm{O}_{3}-\mathrm{Fe}_{2} \mathrm{O}_{3}$ ratios. When comparison is made between the values for magnesia 
determined microscopically and calculated on the assumption of crystalline equilibrium, the differences between the two methods become important when the cooling is more rapid. This is to be expected since no allowance is made for glass or the solubility of magnesia in it when the composition is calculated on the assumption of crystalline equilibrium. The various values obtained for free lime and magnesia are compared graphically in figure 8 ( $A$ and $B$ ).

\section{SUMMARY}

The results of the qualitative and quantitative microscopical examination of 10 samples of commercial portland cement clinker with their slowly and quickly cooled counterparts are reported. These are compared with the quantitative phase composition as obtained from chemical analyses by two methods of calculation to show the deviation to be expected among the different methods.

The general appearance of each phase in the clinker is described together with the changes due to differences which had occurred in heat treatment and cooling rate.

It is shown that there is a definite trend toward simplicity of crystal form and clinker structure as the cooling rate increases. Clinkers cooled in the plant have structures that indicate that their rate of cooling lies intermediate between slow and quick cooling.

Comparisons between calculations of phases from microscopical observations and from chemical analyses, according to the presence or absence of glass, show that:

1. The agreement between phases calculated under the assumption of complete equilibrium crystallization and by the microscopical method is better for the slowly cooled clinkers than for either the plant-cooled or quickly cooled clinkers.

2. In general, for all clinkers, the phase compositions calculated with consideration of glass (normal crystallization) agreed better with the microscopically determined phases than did calculations in which complete equilibrium crystallization was assumed.

3 . The $3 \mathrm{CaO} . \mathrm{SiO}_{2}$ found microscopically nearly always exceeds that calculated by either of the other methods.

4. The $2 \mathrm{CaO} . \mathrm{SiO}_{2}$ microscopically determined was sometimes greater and at other times less than that calculated to be present by either method irrespective of $\mathrm{Al}_{2} \mathrm{O}_{3}-\mathrm{Fe}_{2} \mathrm{O}_{3}$ ratios.

5. The microscopically determined $3 \mathrm{CaO} \cdot \mathrm{Al}_{2} \mathrm{O}_{3}$ was always less than that calculated by either of the other methods.

6. The total dark interstitial phases-rectangular, prismatic, and glass-determined microscopically, approached the total tricalcium aluminate calculated on the assumption of crystalline equilibrium.

7. The $4 \mathrm{CaO} \cdot \mathrm{Al}_{2} \mathrm{O}_{3} \cdot \mathrm{Fe}_{2} \mathrm{O}_{3}$ determined microscopically, in general exceeded that calculated by either of the other methods and showed better agreement for high $\mathrm{Al}_{2} \mathrm{O}_{3}-\mathrm{Fe}_{2} \mathrm{O}_{3}$ ratios than for low.

8. The glass determined microscopically was in better agreement with values obtained from the heat of solution method for slowly cooled and plant-cooled clinkers than for quickly cooled clinkers. 


\section{REFERENCES}

[1] Wm. Lerch and W. C. Taylor, Concrete, Mill Section 45, 199, 217 (1937).

[2] Herbert Insley, E. P. Flint, E. S. Newman, and J. A. Swenson, J. Research NBS 21, 355 (1938) RP1135.

[3] O. A. Radczewski and H. E. Schwiete, Zement 17, 18, 19 (Jan. 1938).

[4] H. Insley and H. F. McMurdie, J. Research NBS 20, 173 (1938) RP1074.

[5] H. Insley, J. Research NBS 17, 353 (1936) RP917.

[6] B. Tavasci, Richerche sulla constituzione del clinker di cemento Portland, Giorn, chim. ind. applicata, 583 (Nov. 1934).

[7] C. K. Wentworth, J. Geol. 31, 228-232 (1923).

[8] H. Insley, J. Research NBS 25, 295 (1940) RP1324.

[9] Olaf Anderson and H. C. Lee, J. Wash. Acad. Sci. 23 [7] (1933).

[10] T. W. Parker and R. W. Nurse, Soc. Chem. Ind. 58, 255 (1939).

[11] H. E. Schwiete and H. zur-Strassen, Zement R3, 511 (1934).

[12] H. F. McMurdie and H. Insley, J. Research NBS 16, 467 (1936) RP884.

[13] L. A. Dahl, Rock Products, 41-42 (1938-1939).

[14] F. M. Lea and T. W. Parker, Dept. Sci. Ind. Res., Bldg. Res. Tech. Paper 16 (1935).

[15] R. H. Bogue, Ind. Eng. Chem., Anal. Ed. 1, 192 (1929).

[16] Wm. Lerch and L. T. Brownmiller, J. Research NBS 18, 617 (1937) RP997.

Washington, November 5, 1940. 